\title{
Increasing reproductive rates of both sexes in dairy cattle breeding optimizes response to selection
}

\author{
C.B. Sagwa, T.O. Okeno ${ }^{\#}$ \& A.K. Kahi \\ Animal Breeding and Genomics Group, Department of Animal Science, Egerton University, \\ P.O. Box 536, 20115, Egerton, Kenya
}

(Received 18 April 2018; Accepted 12 June 2018; First published online 24 July 2019)

\author{
Copyright resides with the authors in terms of the Creative Commons Attribution 4.0 South African Licence. \\ See: http://creativecommons.org/licenses/by/4.0/za \\ Condition of use: The user may copy, distribute, transmit and adapt the work, but must recognise the authors and \\ the South African Journal of Animal Science.
}

\begin{abstract}
It was reasoned that technologies that increase the reproductive rate of males and females in dairy cattle would realize higher responses to selection. The authors tested this hypothesis using deterministic simulation of breeding schemes that resembled those of dairy cattle in Kenya. The response to selection was estimated for four breeding schemes and strategies. Two breeding schemes were simulated, based on artificial insemination (Al) and multiple ovulation and embryo transfer (MOET) reproductive technologies. The strategies were defined according to the use of conventional semen (CS) and X-chromosome-sorted semen (XS). The four strategies therefore were AI with CS (AI-CS) and XS (AI-XS), and MOET with CS (MOET-CS) and XS (MOET-XS). The four strategies were simulated based on the current dairy cattle breeding goal in Kenya. A two-tier closed nucleus breeding programme was considered, with $5 \%$ of the cows in the nucleus and $95 \%$ in the commercial. Dissemination of superior genetic materials in the nucleus was based on all four breeding strategies, while in the commercial only the AI-CS strategy was considered. The strategies that increased the reproductive rates of both males and females (MOET-CS and MOET-XS) realized 2.1, 1.4, and 1.3 times more annual genetic gain, return and profitability per cow, per year, respectively, than strategies that increased the reproductive rates only of males (AI-CS and Al-XS). The use of CS or XS, however, did not affect response to selection in the two schemes. The findings demonstrate that reproductive technologies such as MOET maximize response to selection in dairy cattle breeding.
\end{abstract}

Keywords: artificial insemination, conventional semen, deterministic simulation, multiple ovulation and embryo transfer, $\mathrm{X}$-chromosome-sorted semen

${ }^{\#}$ Corresponding author: otieno24@gmail.com

\section{Introduction}

Dairy cattle production plays an important economic role at household and national level in the tropics (Smith et al., 2013). It is the most vibrant sector in the livestock industry in Kenya (Bebe et al., 2017), and supports over 1.8 million smallholder farmers, who own one to three cows, and account for $70 \%$ and $80 \%$ of the milk produced and marketed, respectively (KDB, 2015). In the recent past there has been a rise in demand for dairy products in Kenya (Bingi \& Tondel, 2015). This has been attributed to an increase in human population, urbanization, increased income per household and greater demand for milk products in East African countries (Bingi \& Tondel, 2015). This demand could be met only through good management of dairy animals and breeding. Improvement through breeding requires an efficient and sustainable breeding programme that accounts for the needs of stakeholders in the dairy cattle value chain. In Kenya, the breeding programme for dairy cattle is well structured as large-scale farms represent the nucleus, and smallholder farms form the lower tier. Although the efficiency of this programme has been assessed based on the current breeding goal, the most effective way to disseminate genetic materials that are generated in the nucleus to smallholder farmers has not been investigated.

Reproductive technologies such as artificial insemination (Al) and multiple ovulation and embryo transfer (MOET) are currently being used with conventional (CS) and sexed semen (XS). Al-CS is the most widely used reproductive technology in Kenya (Omondi et al., 2017). In the recent past, however, most farmers have used MOET and XS (Moore \& Thatcher, 2006; Kosgey et al., 2011), possibly because they are 
interested in maximizing female reproduction and increasing the number of female calves born to overcome the challenge of heifer unavailability. Reproductive technologies could shorten generational intervals, increase selection intensities, and reduce the cost of production, which is attributed to having to transport live breeding candidates from one location to another. Since these technologies increase the reproductive rate of candidates, they would enhance accuracy of selection owing to large numbers of related candidates being recorded. Enhancing the reproductive rate of males and females through Al and MOET, respectively, therefore could result in optimization of response to selection compared with increasing the reproductive rate of only one sex.

Studies have demonstrated that the utilization of reproductive technologies could achieve increased genetic and economic response to selection (Sørensen et al., 2011). Such evaluations, however, are scarce in the tropics. To the authors' knowledge, no study has compared genetic and economic responses to reproductive technologies in Kenya. These technologies were therefore used, based on the assumption that they would yield similar results. The authors used the Kenyan dairy cattle breeding programme as their model to compare the genetic and economic response to selection for reproductive technologies based on $\mathrm{Al}$ and MOET with CS and XS. The evaluation was based on the conventional breeding goal for dairy cattle in Kenya, in which milk sales are based on volume (Kahi et al., 2004). They hypothesized that a combination of the reproductive technologies that increase the reproductive rate of males and females would bring about a higher response to selection than those targeting only one sex.

\section{Materials and Methods}

A deterministic simulation was used to model and estimate the response to selection that is attained by dairy cattle breeding schemes that utilize AI and MOET with CS and XS in a closed two-tier nucleus breeding system. Al-CS was the base scenario with which other strategies were compared. It is the most commonly used reproductive technology among dairy cattle farmers in Kenya. The genetic and economic returns per cow per year for each strategy formed the basis of comparison. In Kenya, two main dairy cattle production systems are recognized, namely commercial and smallholder systems (Onono et al., 2013). Commercial production systems own $20 \%$ of the dairy cattle population and produce $30 \%$ of the marketed milk. They are characterized by owning at least 50 milking dairy cows, and producing their own replacement stock with intensive management regimes (KDB, 2015). The smallholders, on the other hand, own $80 \%$ of the dairy cattle population and produce $70 \%$ of the marketed milk (KDB, 2015). However, they have small pieces of land and cannot produce enough feed and raise heifers. They depend on culled cows and heifers from the commercial farms. This depicts a two-tier closed nucleus system breeding programme as the flow of genetic materials is unidirectional from commercial farms, which act as the nucleus, to smallholder farms, which represent the commercial tier. The breeding programme was developed in such a way that Al and MOET with conventional and sexed semen were used in the nucleus, while only Al-CS was considered in the commercial population. Genetic and economic responses to selection for the breeding strategies were used to compare the schemes.

Development of a breeding goal is the first step in genetic improvement as it defines the direction of selection and genetic merits of performance traits (Åby et al., 2012). It involves i) identifying the breeding, production and marketing systems; ii) identifying sources of income and expenditure; iii) determining the biological traits that influence revenues and costs; and iv) deriving economic values for each trait in the breeding goal. The breeding goal for dairy cattle production in Kenya has been defined (Kahi \& Nitter, 2004). It is market oriented and strives to produce dairy cattle with high milk production under Kenyan conditions. The traits include: milk yield (MY), fat yield (FY), age at first calving (AFC), calving interval (Cl), pre-weaning daily gain (DG), post-weaning daily gain to 18 months (PDG), live weight (LW), pre-weaning survival rate (PreSR), post-weaning survival rate (PostSR) and productive lifetime (PLT). The economic values of these traits were estimated objectively based on change in profitability of the production system due to a unit change in one trait and holding other traits constant. They were estimated under fixed herd and pasture production systems. In each system the economic values were estimated when the price of milk was based on volume or fat content. In this study, the economic values that were estimated under a fixed herd production system were adopted after adjustments to reflect current market inflation rates. In Kenya factors such as land size, labour, management skills and availability of feed determine herd size. The adjustments of the economic values were necessary because the market is dynamic, and input and output prices change over time depending on inflation rates. These economic values were therefore adjusted by multiplying them by their cumulative discounted expressions. Cumulative discounted expressions reflect time and frequency of future expression of a trait in a superior genotype from selected parents (Berry et al., 2006). The traits in the breeding goal, their economic values and genetic parameters are presented in Table 1 . The genetic parameters were obtained from studies conducted in Kenya. Where such values were missing, other studies in the tropics were consulted (Kahi \& Nitter, 2004; Kahi et al., 2004; Ilatsia et al., 2011). 
Table 1 Heritability estimates (along diagonal bold), phenotypic standard deviations, economic values, genetic correlations (above diagonal) and phenotypic correlations (below diagonal) for traits in the breeding goal and selection criteria

\begin{tabular}{lcccccccccc}
\hline & \multicolumn{10}{c}{ Traits } \\
\cline { 2 - 11 } & MY & FY & AFC & Cl & DG & PDG & LW & PreSR & PostSR & PLT \\
\hline $\boldsymbol{\sigma}_{\mathbf{p}}$ & 1208.46 & 36.57 & 448.76 & 75.34 & 19.00 & 743.00 & 54.14 & 30.00 & 30.00 & 864.90 \\
EVs & 16.05 & 79.44 & -2.72 & 2.65 & 1.04 & 3.4 & 7.95 & 9.96 & 45.15 & 0.07 \\
MY & $\mathbf{0 . 3 0}$ & 0.91 & 0.20 & 0.17 & 0.10 & 0.11 & 0.23 & 0.00 & 0.00 & 0.00 \\
FY & 0.73 & $\mathbf{0 . 3 2}$ & -0.10 & 0.08 & 0.10 & 0.11 & 0.12 & 0.00 & 0.00 & 0.00 \\
AFC & -0.21 & 0.05 & $\mathbf{0 . 3 8}$ & -0.21 & -0.20 & -0.20 & 0.15 & 0.00 & 0.00 & -0.13 \\
CI & 0.17 & 0.08 & -0.21 & $\mathbf{0 . 0 6}$ & 0.00 & 0.00 & -0.40 & 0.00 & 0.00 & 0.10 \\
DG & 0.10 & 0.10 & -0.25 & 0.10 & $\mathbf{0 . 2 9}$ & -0.25 & 0.20 & 0.06 & 0.03 & 0.10 \\
PDG & 0.11 & 0.11 & -0.25 & 0.10 & 0.49 & $\mathbf{0 . 3 2}$ & 0.25 & 0.03 & 0.06 & 0.10 \\
LW & 0.23 & 0.12 & 0.15 & -0.43 & 0.40 & 0.47 & $\mathbf{0 . 3 0}$ & 0.01 & 0.00 & 0.27 \\
PreSR & 0.00 & 0.00 & 0.00 & 0.00 & 0.06 & 0.03 & 0.01 & $\mathbf{0 . 0 9}$ & 0.00 & 0.00 \\
PostSR & 0.00 & 0.00 & 0.00 & 0.00 & 0.03 & 0.06 & 0.00 & 0.01 & $\mathbf{0 . 0 9}$ & 0.00 \\
PLT & 0.00 & 0.00 & -0.13 & 0.10 & 0.10 & 0.10 & 0.27 & 0.00 & 0.00 & $\mathbf{0 . 1 1}$ \\
& & & & & & & & & & \\
\hline
\end{tabular}

MY: milk yield (kg); FY: fat yield (kg); AFC: age at first calving (days); Cl: calving interval (days); DG: pre-weaning daily gain (g/day); PDG: post-weaning daily gain to 18 months (g/day); LW: live weight (kg); PreSR: pre-weaning survival rate (\%); PostSR: post-weaning survival rate (\%); PLT: cow productive lifetime (days)

$\sigma_{\mathrm{p}}$ : phenotypic standard deviation

EV: economic values

(Source: Kahi et al., 2004; Kahi \& Nitter 2004; Ilatsia et al., 2011)

A two-tier closed nucleus breeding system was considered. This system assumed a single direction of flow of genetic material in which the genetic gain that was generated in the nucleus was disseminated to the commercial population. This implies that the nucleus does not allow genetic materials to be introduced from outside. The genetically superior candidates were therefore retained in the nucleus as replacement stock, while the remaining candidates were sold to the commercial tier for breeding. Four strategies were considered, based on the reproductive technology adopted in the breeding programme:

Artificial Insemination with conventional semen (Al-CS): This strategy assumes that Al was the only reproductive technology used to disseminate genetic materials in the nucleus. In this strategy only CS was considered. This strategy represent the most commonly used reproductive technology in dairy breeding in Kenya currently.

Artificial Insemination with X-sorted semen (Al-XS): This strategy is similar to Al-CS, but only XS was used to inseminate cows in the nucleus. The use of this strategy is currently increasing in Kenya, especially in large-scale dairy farms.

Multiple ovulation and embryo transfer with conventional semen (MOET-CS): This strategy increases the reproductive rate of both males and females. It involves stimulating a donor cow to release many ova from its ovaries, which are then fertilized with conventional semen. Thereafter, the embryos develop and can be flushed from the uterus of the donor cow and transplanted to heat-synchronized recipient cows that carry the pregnancy to term. Although this strategy is not common in Kenya, it is being practised on some largescale farms.

Multiple ovulation and embryo transfer with X-sorted semen (MOET-XS): This strategy is similar to MOET-CS, but the oocytes are fertilized with XS.

A simulated population of 50000 cows was distributed between the two tiers. The top tier (nucleus) consisted of $5 \%$ of the highest ranking cows in the population, while the remaining $95 \%$ constituted the lower tier (commercial population). The biological and economic parameters used in the current study were obtained from previous studies on dairy cattle in Kenya (Kahi \& Nitter, 2004; Kahi et al., 2004; Ilatsia et al., 2011). Truncation selection based on estimated breeding values was used to select top ranking males and females for breeding in the nucleus. The second top ranking males and females were used for breeding in the lower tier. The young bull scheme was used to disseminate genetic materials in the population. The use 
of the young bull scheme was recommended because of the short generation interval (Kahi et al., 2004). Candidates that were not selected for breeding in the nucleus and commercial populations were culled and sold for meat production. AI-CS, AI-XS, MOET-CS and MOET-XS were used in the nucleus, and only AI-CS was considered in the commercial population.

Table 2 Population, biological and technical details on nucleus and commercial herds of cattle used in study

\begin{tabular}{ccc}
\hline Population information & Nucleus & Commercial \\
Number of cows & 2500 & 47500 \\
Proportion of cows in nucleus and commercial (\%) & 5 & 95
\end{tabular}

\section{Productive life time (years)}

Bulls to breed bulls in nucleus

Dams in the nucleus to breed bulls in the nucleus 5

Bulls in the nucleus to breed dams in the nucleus 5

Dams in the nucleus to breed dams in the nucleus 7

Bulls in the nucleus to breed bulls in the commercial 5

$\begin{array}{ll}\text { Dams in the commercial to breed bulls in the commercial } & 7\end{array}$

Bulls in the commercial to breed dams in the commercial 3

\section{Age at first calving (years)}

Bulls to breed bulls in the nucleus 2

Dams in the nucleus to breed bulls in the nucleus 3

Bulls in the nucleus to breed dams in the nucleus 2

Dams in the nucleus to breed dams in the nucleus 3

Bulls in the nucleus to breed bulls in the commercial 2

Dams in the commercial to breed bulls in the commercial 4

Bulls in the commercial to breed dams in the commercial 3.5

\section{Additional measurements}

$\begin{array}{lcc}\text { Pre-weaning survival rate (\%) } & 0.99 & 0.90 \\ \text { Post-weaning survival rate (\%) } & 0.99 & 0.92 \\ \text { Calving interval (years) } & 1.30 & 1.30 \\ \text { Proportion of sires suitable for breeding (\%) } & 0.89 & 0.80 \\ \text { Proportion of cows suitable for breeding (\%) } & 0.90 & 0.81 \\ \text { Number of calves per cow per year in Al strategy } & 0.95 & 0.60 \\ \text { Number of calves per cow per year for MOET strategy } & 10 & 0.00 \\ \text { Number of cows per bull } & 500 & 500 \\ \text { Replacement rate of cows per year (\%) } & 0.25 & 0.25 \\ \text { Number of inseminations per conception using conventional semen } & 2.00 & 2.00 \\ \text { Number of inseminations per conception using sexed semen } & 3.00 & 0.00 \\ \text { Probability of getting a heifer when using sexed semen } & 0.90 & 0.00 \\ \text { Probability of getting a heifer when using conventional semen } & 0.50 & 0.50\end{array}$

Al: artificial insemination; MOET: multiple ovulation and embryo transfer

The mating ratio was one bull to 100 cows in both the nucleus and commercial systems. The number of calves per cow per year in Al strategies was assumed to be 0.95 and 0.60 in the nucleus and commercial, respectively. In the MOET strategies, each cow in the nucleus was assumed to produce 10 calves per year. The sex ratio for strategies using CS was 0.5 , while the male to female ration for those using XS was 
$0.05: 0.95$. Two and three inseminations per conception were assumed for use of CS and XS, respectively. Various selection pathways were considered in disseminating genetic gain. The main pathways were sires to breed sires (SS) and dams (SD), and dams to breed sires (DS) and dams (DD). Each selection group had different sources of information for traits in the breeding goal. The information sources for SS and SD were records of individual, sire, dam, dams of the sire and dam, while those for DS and DD were records of the individual, dam, sire, all female paternal half sibs of the dam and sire, dams of the sire and dam. The input populations, biological and technical parameters that were used to model the breeding schemes are presented in Table 2.

The breeding system was initiated by sampling unrelated base populations of bulls and cows. There were 2500 cows in the nucleus and 47,500 in the commercial. Five hundred bulls were used in both the nucleus and commercial. Semen was collected from the bulls. Half was used as CS and the other half was sorted for X-chromosome, prior to use. For each animal $\boldsymbol{i}$ in the base population, a vector of true breeding values $\left(\mathrm{tbv}_{\mathrm{i}}\right)$ was calculated for all simulated traits using the following equation:

$$
t b v_{i}=L^{\prime *} r_{1}
$$

where: $\mathbf{L}^{\prime}$ is the Cholesky decomposition of the (co)variance matrix $\mathbf{G}$, and $\mathbf{r}_{\mathbf{l}}$, a vector of random numbers from standardized normal distribution.

In later generations $\mathbf{t b v}_{\mathbf{i}}$ was simulated as;

$$
t b v_{i}=0.5 *\left(t b v_{i(\text { sire })}-t b v_{i(d a m)}\right)
$$

The phenotypes of the traits for the $i^{\text {th }}$ based animal were calculated as;

$$
o b s_{i}=t b v_{i}+c^{\prime *} r
$$

where: C' is the Cholesky decomposition of the environmental (co)variance matrix $\mathbf{R}$, and $\mathbf{r}$ is a vector of random numbers from a standardized normal distribution.

All the breeding values were predicted using best linear unbiased prediction (BLUP) by fitting a multivariate animal model to the phenotypes. The model was computed as:

$$
\mathbf{y}=\mathbf{X b}+\mathbf{Z} \mathbf{a}+\mathbf{e}
$$

where: $\mathbf{Y}$ is the vector of phenotypes, a vector of fixed effects,

$a$ is a vector of random animal effects,

e, a vector of residual errors, and

$\mathbf{X}$ and $\mathbf{Z}$ the incidence matrices.

The breeding values were computed using (co)variance matrix presented below:

$$
\left(\begin{array}{l}
\mathbf{a} \\
\mathbf{e}
\end{array}\right) \sim \mathrm{N}\left(0 ;\left[\begin{array}{cc}
\mathrm{G} \otimes \mathrm{A} & 0 \\
0 & \mathrm{R} \otimes \mathrm{I}
\end{array}\right]\right)
$$

where: the matrix $\mathbf{A}$ is the numerator relationship matrix among all animals, the matrix $\mathbf{G}$ is the additive genetic (co)variance matrix of traits in the breeding goal, and the matrix $\mathbf{R}$ is the (co)variance matrix for residual effects.

The economic returns were determined based on profitably per cow in each breeding system. The profitability per cow was estimated as: 


$$
\pi=\sum_{t=0}^{T}\left(\frac{R_{t}-c_{t}}{(1+r)^{t}}\right)
$$

where: $T$ is the evaluation period (25 years),

$R_{t}$ the annual benefits of genetic improvement calculated as realized genetic gain per cow per year, $c_{t}$ the costs of genetic improvement which includes fixed and variable costs and $r$ the discounting rate.

The discounting rate of $5 \%$ was recommended when evaluating animal breeding programmes (Bird \& Mitchel, 1980) and was adopted in the current study. Variable costs are presented in Table 3. These included costs that were directly related to performance and pedigree recording. Fixed costs were those incurred in one round of selection and were the overhead costs of running the nucleus of 2,500 cows. The average time at which fixed costs occurred was assumed to be the mean generation interval. Variable and fixed costs affect only the profit, and not the genetic response. The interest rates for returns (8\%) and costs (6\%) were based on the current marketing conditions in Kenya (CBK, 2017).

Table 3 Variable costs in the nucleus in Kenya shillings

\begin{tabular}{lc}
\hline & $\begin{array}{c}\text { Costs } \\
\text { (KES) }\end{array}$ \\
\hline Variable costs & \\
Identification, pedigree recording and data processing & 105.00 \\
Milk recording & 30.00 \\
Fat analysis and recording & 100.00 \\
Age at first calving recording & 30.00 \\
Calving Interval recording & 30.00 \\
Daily gain recording & 30.00 \\
Post weaning daily gain recording & 30.00 \\
Bull assessment & 200.00 \\
Cows assessment & 200.00 \\
Conventional semen per straw & 1000.00 \\
Sexed semen per straw & 5000.00 \\
Cows synchronisation, embryo collection and transfer & $50,000.00$ \\
Labour & 0.14 \\
\hline
\end{tabular}

Kenya shillings $100=1$ USD

The responses to selection in all the simulated breeding strategies were evaluated in terms of annual genetic gain, return on investment and profitability after an investment period of 25 years. Genetic gain was calculated per cow per year. The rate of genetic gain for each cow was predicted as linear regression of true breeding values for each trait in the breeding goal weighted by its corresponding economic values and expressed per year. Profitability was computed as the difference between the returns and the total costs.

A deterministic computer programme for simulating livestock breeding programmes, namely ZPLAN z10 (Willam et al., 2008), was used to model and evaluate the breeding systems. Using the gene flow methods and selection index procedures, ZPLAN simulates various breeding plans in any livestock species. It computes genetic gain for the aggregate breeding value, the annual response for each selection and correlated trait and the profit per female animal in the population by subtracting breeding costs from returns. The programme uses the genetic, biological and economic measurements provided in the input files to calculate the costs and returns. The calculations assume that the input measurements and selection strategies remain unchanged over the investment period with one round of selection. Reduction in genetic variance and change in rate of inbreeding, however, is not considered. The programme applies order statistics to obtain adjusted selection intensities for population with finite sizes. ZPLAN has been used widely 
to model and evaluate cattle breeding programmes such as those for dairy cattle (Kahi et al., 2004), dualpurpose cattle (llatsia et al., 2011) and beef cattle (Rewe et al., 2010).

\section{Results}

The findings of the current study confirmed the hypothesis that breeding schemes that utilize technologies that increase the reproductive rates of males and females realize a higher response to selection compared with those that increase the reproductive rates of only one sex. The responses to selection based on annual genetic gain, returns, costs and profit of these strategies after the investment period of 25 years are presented in Table 4.

Table 4 Annual genetic gain, returns, costs per cow for four breeding strategies after 25 years

\begin{tabular}{lcccc}
\hline & \multicolumn{3}{c}{ Strategies } \\
\cline { 2 - 5 } & Al-CS & Al-XS & MOET-CS & MOET-XS \\
\hline Genetic gain & 143.97 & 143.97 & 301.42 & 301.42 \\
Returns per cow & 1046.51 & 1046.51 & 1951.06 & 1951.06 \\
Cost per cow & 53.67 & 85.21 & 181.15 & 212.71 \\
Profit per cow & 992.84. & 961.30 & 1769.91 & 1738.35 \\
\hline
\end{tabular}

Al-CS: artificial insemination with conventional semen; Al-XS: artificial insemination with X-chromosome-sorted semen; MOET-CS: multiple ovulation and embryo transfer with conventional semen; MOET- XS: multiple ovulation; embryo transfer X-chromosome-sorted semen

Genetic gain and profitability were affected by the reproductive technology, whereas the use of CS and XS affected only the costs and profitability of the breeding programme. The strategies that increase the reproductive rates of males and females (MOET-CS and MOET-XS) realized 2.1, 1.8, and 1.8 times more annual genetic gain, return and profitability per cow, respectively, compared with those that increased only the reproductive rates of males (AI-CS and AI-XS). The cost per cow per year for MOET-CS and MOET-XS, however, was 3.4-fold and 2.5-fold higher than those realized in AI-CS and Al-XS, respectively. Although the use of CS and XS did not have an effect on annual genetic gain and return per cow when used with Al or MOET, they affected the costs and profitability per cow per year. The Al-XS and MOET-XS strategies realized additional costs of KES 31.54 compared with AI-CS and MOET-CS strategies. The corresponding profitability per cow per year was therefore reduced by a similar amount.

Table 5 Annual genetic gain in individual traits in the four scenarios using various reproductive technologies

\begin{tabular}{lcc}
\hline \multirow{2}{*}{ Trait } & \multicolumn{2}{c}{ Breeding strategies } \\
\cline { 2 - 3 } & Al-CS & MOET-CS \\
\hline Milk yield & 6.97 & 20.04 \\
Fat yield & 0.35 & 0.77 \\
Age at first calving & -1.12 & -2.54 \\
Calving Interval & 0.02 & 0.11 \\
Average daily gain & 0.01 & -0.01 \\
Pre-weaning daily gain & 1.69 & 4.57 \\
Live weight & 0.06 & 0.23 \\
Cow productive life time & 0.16 & 1.20 \\
\hline
\end{tabular}

Al-CS: artificial insemination with conventional semen

MOET-CS: multiple ovulation and embryo transfer with conventional semen 
The response to selection for individual traits in the breeding goal followed the same trend that was observed in returns to selection. Traits in the breeding goal for the schemes using strategies that increased reproductive rates of both males and females (MOET based) realized higher genetic gains than those that were Al based (Table 5). The use of CS and XS, however, did not have an effect on response to selection for the individual traits and therefore only the findings from AI-CS and MOET-CS are presented in Table 5.

Productive traits such as milk yield, fat yield, daily gain, pre-weaning daily gain and live weight realized additional 13.07, 0.42, 0.02, 2.88 and $0.170 \mathrm{~kg}$, respectively, in the MOET-CS compared with Al-CS schemes. On the other hand, the reproductive and longevity traits realized an increase in response to selection. Age at first calving was reduced by 1.42 days and calving interval increased by 0.09 days. Cow productive lifetime increased by 1.04 days.

The intensity and accuracy of selection for the estimated breeding values in the Al-CS and MOET-CS are presented in Table 6. As expected, this was affected by the reproductive technology and sex of the selection candidates. The reproductive technology that increased the reproductive rate of both sexes (MOET-CS) realized an additional 0.21 and 0.61 in selection intensity of males and females, respectively, compared with AI-CS. The corresponding increase for accuracy of selection was $10 \%$ and $11 \%$. The males generally realized higher intensity compared with females in both breeding strategies. However, females outperformed males in accuracy of selection.

Table 6 Intensity and accuracy of selection for males and females in the breeding schemes

\begin{tabular}{lcccc}
\hline \multirow{2}{*}{ Breeding strategies } & \multicolumn{2}{c}{ Selection Intensity } & \multicolumn{2}{c}{ Accuracy of selection } \\
\cline { 2 - 5 } & Males & Females & Males & Females \\
\hline \multirow{2}{*}{ Al-CS } & 2.34 & 1.46 & 0.53 & 0.66 \\
MOET-CS & 2.55 & 2.07 & 0.63 & 0.77 \\
\hline
\end{tabular}

AI-CS: artificial insemination with conventional semen

MOET-CS: multiple ovulation and embryo transfer with conventional semen

\section{Discussion}

The findings of this study support the hypothesis that increasing the reproductive rate of both males and females would optimize response to selection in dairy cattle. These findings are supported by Kosgey et al. (2005) and Pedersen et al. (2012), who demonstrated that the adoption of MOET breeding schemes realized a higher response to selection compared with Al schemes using either CS or XS in dairy cattle. This implies that irrespective of the semen used to inseminate the cows (conventional or sexed), increasing the number of offspring per cow has a major impact on total genetic gain and monetary returns. The superiority of MOET over Al breeding schemes was attributed to intensity and accuracy of selection. The contribution of selection intensity has two possible explanations. First, intensity of selection has a direct impact on repose to selection. Therefore any breeding strategy that increases the number of candidates for selection realizes a higher response to selection. This was evident in the current study as the number of candidates from which to select increased from one to 10 calves per cow per year in the MOET breeding schemes. Since the number of selection candidates was constant throughout the simulation period, there were more proven candidates from which to select. This is reflected in the current study as MOET schemes had $21 \%$ and $61 \%$ increased intensity to selection in males and females, respectively, compared with Al schemes. Secondly, the intensity of selecting males was higher than those of females in both MOET and Al schemes. This is because fewer males are needed for breeding compared with females. The high intensity of selecting males was reported in other studies (Kosgey et al., 2005; Pedersen et al., 2012; Granleese et al., 2015). MOET technology enables each cow to produce more offspring per year, thus increasing the number of selection candidates. This results in high selection intensity and therefore higher response to selection compared with $\mathrm{Al}$, which increases the reproductive rate only of males. Increased accuracy of selection in the MOET-CS scheme compared with Al-CS could be attributed to the higher number of offspring per cow. Each cow produced 10 calves per year. These offspring were closely related to the selection candidates and therefore provided the information to compute their breeding values, thus increasing response to selection. Increasing the number of phenotypes increases accuracy of selection and therefore response to selection in breeding programmes (Dekkers, 2004). The non-differences in genetic gain and returns per cow that were observed in the breeding goal when conventional or sexed semen were used in Al and MOET schemes were confirmed 
by the response for individual traits (Table 5). These findings partially agree with those reported by Sørensen et al. (2011). In that study, the use of sexed semen with MOET was found to marginally increase the response to selection, although it was not significantly different from zero. The current findings could be attributed to the higher contribution of males to genetic gain than females. This is in line with previous studies that compared the contributions of males and females to response to selection (Henryon et al., 2012; Okeno et al., 2014). In these studies, response to selection was found to be optimized when males were genotyped and phenotyped. The current study therefore demonstrates that the superiority of MOET over Al in response to selection could be attributed to increased intensity and accuracy of selection.

Although no differences were observed in response to genetic gain and returns per cow when CS and XS were used in Al and MOET breeding schemes, the costs were higher in schemes that used XS (Table 4). This contributed to the low profitability realized in AI-XS and MOET-XS in the current study. The high costs in schemes that use XS could be explained by the high price of XS in Kenya, because of the marketing narrative by semen companies that XS produces superior breeding stock compared with CS. This narrative has been disapproved in the current study. The combination of MOET-XS was less attractive as it realized low profitability. This implies that the utilization of MOET-CS would be more attractive in the genetic and economic responses under Kenyan production conditions.

The implementation of MOET-CS, however, may remain a challenge for two reasons. First, the scheme requires a well-established infrastructure and trained personnel for implementation, which are still inadequate (Kosgey et al., 2006; Van Arendonk, 2011). Second, the high costs of synchronizing the cows, semen importation and technician services pose challenges. This was evident in the current study as MOET schemes were 3.40 times more expensive than Al schemes. This implies that most of the smallholder farmers, who own $80 \%$ of dairy cattle in Kenya (KDB, 2015), would not be able to participate in this scheme. MOET-CS may be feasible only with government intervention in subsidies. Since the Kenyan dairy sector is completely liberalized (KDB, 2015), subsidies should not be expected by dairy farmers in the near future. Therefore, Al schemes may continue to play significant role in the genetic improvement of dairy cattle.

\section{Conclusion}

The findings of this study confirmed that breeding schemes such as MOET that increase the reproductive rates of both males and females maximize response to selection. It also demonstrated that although genetic gain and returns per cow per year were not influenced by the use of conventional or sexed semen, sexed semen influenced the costs and therefore the profitability of the breeding programme. Adoption of a breeding strategy that uses CS with MOET would be more beneficial to dairy cattle farmers in Kenya if the necessary infrastructure is put in place for smooth operation of the breeding programme.

\section{Acknowledgements}

The authors would like to thank iLINOVA and Egerton University for provision of facilities.

\section{Authors' Contributions}

CBS participated in design of the study, did data analysis and manuscript writing. TOO was involved in the design of the study, modelling statistical analysis and manuscript writing. AKK was involved in modelling, interpreting the findings and revising the manuscript.

\section{Conflict of Interest Declaration}

The authors declare that they have no competing interests.

\section{References}

Åby, B.A., Aass, L., Sehested, E. \& Vangen, O., 2012. A bio-economic model for calculating economic values of traits for intensive and extensive beef cattle breeds. Livest. Sci. 143, 259-269.

Bebe, B.O., Rademaker, C.J., Van der Lee. J., Kilelu, C.W. \& Tonui, C., 2017. Sustainable growth of the Kenyan dairy , Wageningen Livest. Res. 1021, 1-6.

Berry, D.P., Madalena, F.E., Cromie, A.R. \& Amer, P.R., 2006. Cumulative discounted expressions of dairy and beef traits in cattle production systems. Livest. Sci. 99, 159-174.

Bingi, S. \& Tondel, F., 2015. Recent developments in the dairy sector in Eastern Africa: Towards a regional policy framework for value chain development. European Centre for Development Policy Management. Briefing Note, 78.

Bird, P.J. \& Mitchell, G., 1980. The choice of discount rate in animal breeding investment appraisal. Anim. Breed. Abstr. 48, 499-505.

CBK, 2017. Central Bank of Kenya. Available at: http://www.cbk.go.ke. Accessed 4 January 2018.

Dekkers, J.C. 2004. Commercial application of marker-and gene-assisted selection in livestock: Strategies and lessons 1 2. J. Anim. Sci. 82, 313-328.

Granleese, T., Clark, S.A., Swan, A.A. \& Werf, J.H. 2015. Increased genetic gains in sheep, beef and dairy breeding programs from using female reproductive technologies combined with optimal contribution selection and genomic 
breeding values. Genet. Select. Evolution 47, 70-83

Henryon, M., Berg, P., Ostersen, T., Nielsen, B. \& Sørensen, A.C., 2012. Most of the benefits from genomic selection can be realized by genotyping a small proportion of available selection candidates. J. Anim. Sci. 90, 4681-4689.

Ilatsia, E.D., Roessler, R., Kahi, A.K., Piepho, H.P. \& Zárate, A.V., 2011. Evaluation of basic and alternative breeding programs for Sahiwal cattle genetic resources in Kenya. Anim. Prod. Sci. 51, 682-694.

Kahi, A.K. \& Nitter, G., 2004. Developing breeding schemes for pasture based dairy production systems in Kenya: I. Derivation of economic values using profit functions. Livest. Prod. Sci. 88, 161-177.

Kahi, A.K., Nitter, G. \& Gall, C.F., 2004. Developing breeding schemes for pasture based dairy production systems in Kenya: II. Evaluation of alternative objectives and schemes using a two-tier open nucleus and young bull system. Livest Prod. Sci. 88, 179-192.

KDB, 2015. Kenya Dairy Board. http://www.kdb.go.ke. Accessed on 11 November, 2016.

Kosgey, I.S., Kahi, A.K. \& Van Arendonk, J.A.M., 2005. Evaluation of closed adult nucleus multiple ovulation and embryo transfer and conventional progeny testing breeding schemes for milk production in tropical crossbred cattle. J. Dairy Sci. 88, 1582-1594.

Kosgey, I.S., Baker, R.L., Udo, H.M.J. \& Van Arendonk, J.A.M., 2006. Successes and failures of small ruminant breeding programmes in the tropics: A review. Small Rumin. Res. 61, 13-28.

Kosgey, I.S., Mbuku, S.M., Okeyo, A.M., Amimo, J., Philipsson, J. \& Ojango, J.M., 2011. Institutional and organizational frameworks for dairy and beef cattle recording in Kenya: A review and opportunities for improvement. Anim. Gen. Res. 48, 1-11.

Moore, K. \& Thatcher, W.W., 2006. Major advances associated with reproduction in dairy cattle. J. Dairy Sci. 89, 1254-1266.

Okeno, T.O., Henryon, M. \& Sørensen, A.C., 2014. Benefits of distributing males and females among phenotyping candidates in genomic selection. In: Book of abstracts of 65th Annual Meeting of the European Federation of Animal Science 20, 215-215.

Omondi, I.A., Zander, K.K., Bauer, S. \& Baltenweck, I., 2017. Understanding farmers' preferences for artificial insemination services provided through dairy hubs. Animal 11, 677-686.

Onono, J.O., Wieland, B. \& Rushton, J., 2013. Productivity in different cattle production systems in Kenya. Trop. Anim. Health Prod. 45, 423-430.

Pedersen, L.D., Kargo, M., Berg, P., Voergaard, J., Buch, L.H. \& Sørensen, A.C., 2012. Genomic selection strategies in dairy cattle breeding programmes: Sexed semen cannot replace multiple ovulation and embryo transfer as superior reproductive technology. J. Anim. Breed. Genet. 129, 152-163.

Rewe, T.O., Herold, P., Piepho, H.P., Kahi, A.K. \& Zárate, A.V., 2010. Genetic and economic evaluation of a basic breeding programme for Kenya Boran cattle. Trop. Anim. Health Prod. 42, 327-340.

Smith, J., Sones, K., Grace, D., MacMillan, S., Tarawali, S. \& Herrero, M., 2013. Beyond milk, meat, and eggs: Role of livestock in food and nutrition security. Anim. Front. 3, 6-13.

Sørensen, MK., Voergaard, J., Pedersen, L.D., Berg, P. \& Sørensen, A.C., 2011. Genetic gain in dairy cattle populations is increased using sexed semen in commercial herds. J. Anim. Breed. Gen. 12, 267-275.

Van Arendonk, J.A.M., 2011. The role of reproductive technologies in breeding schemes for livestock populations in developing countries. Livest. Sci. 136, 29-37.

Willam, A., Nitter, G., Bartenschlager, H., Karras, K., Niebel, E. \& Graser, H.U., 2008. ZPLAN, A computer program to optimise livestock selection schemes. User guide for ZPLAN 2007 (Z10). Hohenheim University, Stuttgart, Germany. 